\title{
Patient's Perception of Health Information Security: The Case of Selected Public and Private Hospitals in Addis Ababa
}

\author{
Tibebe Beshah \\ Information Science, Addis Ababa University \\ Addis Ababa, Ethiopia \\ tibebe.beshah@gmail.com
}

\author{
Dawn Medlin \\ Computer Information Systems, Appalachian State \\ University \\ Boone, North Carolina \\ medlinbd@appstate.edu
}

\author{
Ajith Abraham \\ Machine Intelligence Research Labs (MIR Labs), Scientific Network for Innovation and Research Excellence, USA \\ ajith.abraham@ieee.org
}

\begin{abstract}
Information security in health sector is getting growing attention. In this connection, patient's perception about different aspects of health sector is worth considering. In this research, attempt has been made to assess and analyze patient's perception of health information security at some selected public and private hospitals in Addis Ababa, Ethiopia. Quantitative research approach using questionnaire as an instrument was employed in an attempt to empirically address the topic. The research result reveals that patient's perception of health information security is generally low. Major determinant factors for their perception include their educational background, age and general awareness. It is also worth mentioning that patient's perception has strong implication on the service delivery and satisfaction of both service providers and patients themselves
\end{abstract}

Keywords-Information security, Patients perception, Public Hospitals, Private Hospitals, Ethiopia

\section{INTRODUCTION}

Security in general and information security in particular is an issue of growing importance and when it is in the area of health it is more serious. As part of the various efforts in the area, research works related to privacy, security and trust are getting more attention. In line with this, through this study, attempt is being made to assess patient's perception of health information security at some selected public and private hospitals in Ethiopia.

Thus the rest of this research paper is structured as follows. This Section presents the theoretical background of the study, objective, rationale, significances, and research questions. The second Section contains detailed methodological and procedural descriptions. Results and discussions of the findings are also presented in the third Section followed by conclusions and recommendations.

\section{A. Theoretical background}

It is widely accepted that most of human activities demand the availability, access and use of relevant information. According to Willison et al. (2003) information is useful for decision-making only if it is collected, processed, stored and used properly and ethically. Information is created in different organizations for different purposes. Health institutions are among these organizations to collect, process, store, and use health related information. In addition to diagnostic and testing information, the medical record includes the details of a person's family history, genetic testing, history of diseases and treatments, history of drug use, sexual orientation and practices, and testing for sexually transmitted disease. Subjective remarks about a patient's demeanor, character, and mental state are also parts of the record.

As stated by Mercuri (2004), through time, a patient's medical record accumulates significant personal information including identification, history of medical diagnosis, digital renderings of medical images, treatment received, medication history, dietary habits, sexual preference, genetic information, psychological profiles, employment history, income, and physicians" subjective assessments of personality and mental state among others. Thus it is easy to learn that, health information is primarily an essential part of a patient's present and future health care in addition to its use in the management and planning of healthcare facilities and services, medical training and research and the production of healthcare statistics. It is also stated by the Legislative Reference Bureau of Hawaii (2000) that, since health information has the potential both to improve health and to do harm, organizations and individuals that create, use and provide health information should be trustworthy, protect patient health information and adhere to security principles.

Information security in health sector refers to protecting personal health related records from unauthorized access, use, disclosure, disruption, modification or destruction. Patients fear that their personal medical information may influence their employers' decisions about promotions or downsizing or be made public in press reports or civil court actions. There might be various forms of information security threats at health sectors. in connection to this threats to patient privacy and information security could be categorized into two broad areas: (1) Organizational threats that arise from inappropriate access of patient data by either internal agents abusing their privileges or external agents exploiting vulnerability of information systems, and (2) Systemic threats that arise from an agent in the information 
flow chain exploiting the disclosed data beyond its intended use (NRC 1997; Appari and Johnson, 2008).

As cited by Appari and Johnson, (2008), a survey of researches on health care confidentiality made by Sanker et al. (2003) revealed four overarching conclusions. First, patients strongly believe that their information should be shared only with people involved in their care. Second, patients do identify with the need of information sharing among physicians, though HIV patients are less likely to approve sharing of their health information. Third, many patients who agree to information sharing among physicians reject the notion of releasing information to third parties including employers and family members. Lastly, the majority of patients who have undergone genetic testing believe that patients should bear the responsibility of revealing test results to at-risk family members. In the same line it is stated that very limited research has examined patients' perception on sharing of anonymized health records, perhaps 10 with exception of more recent studies that examine patients perception about consent to health information use for other than their own care (Bansal, et al. 2007; Campbell et al. 2007).

Being one of a few attempts in studying patients perception, Bansal et al. (2007), reported that user's current health status, personality traits, culture, and prior experience with websites and online privacy invasions play a major role in user's trust in the health website and their degree of security and privacy concerns. Campbell et al. (2007) also showed that significant numbers of patients are neutral to their health related information disclosure. In connection to this, Angst et al. (2006) investigated divergence of perception among patients toward different types of personal health record systems, including paper based, personal computer based, memory devices, portal and networked PHR, which are in the increasing order of technological advancement. The study found that patient's relative perception of privacy and security concern increased with the level of technology, e.g. relative security and privacy concern for networked PHR is twice that of memory device based PHR. Technologically advanced PHR systems were found to be favored by highly educated patients.

In another study, Chalmers and Muir (2003) stated that, patients should have the right to exercise some control over the acquisition, use, and disclosure of their personal health information. In connection to this their perception towards it, will have a positive or negative implication on service delivery and user satisfaction. Security and privacy (information) are also parts of the basic human rights clearly stated in different constitutions, civil and criminal codes and regulations.

The medical record is the primary source for much of the health care information sought by parties inside and outside the direct health care delivery services, such as prescription and drug use, treatment outcomes, and reason for and length of hospital stay. These data are important because health care information can influence decisions about an individual's access to appropriate health care, credit, admission to educational institutions, and his or her ability to secure employment and obtain insurance. Its improper disclosure, can deny an individual access to these basic necessities of life, and can threaten an individual's personal and financial well-being. Many believe that the efficacy of the healthcare relationship depends on the patient's understanding that the information recorded by a physician will not be disclosed. Many patients might refuse to provide physicians with certain types of information needed to render appropriate care if patients do not believe that information would remain confidential.

As indicated in Figure 1, there are a number of research domains in information security in the health sector. Researches from patient's perspective, public-policy related, inter-organizational productivity and quality are the major ones. In the same line this specific research will fall under the first category of research domains of information security identified by Appari and Johnson (2008).

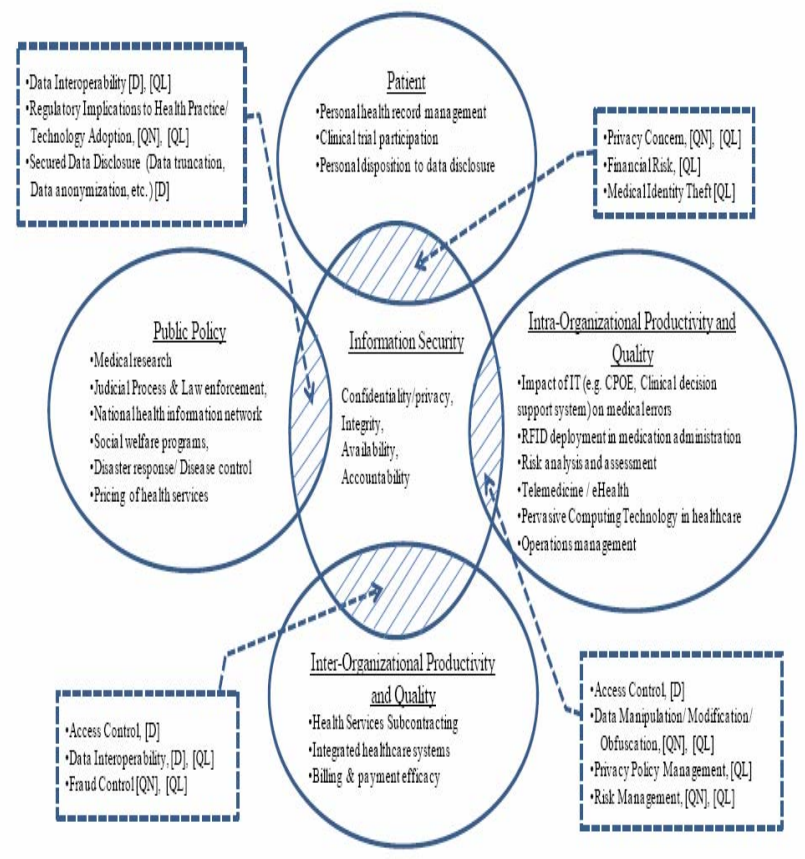

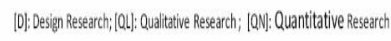

FIGURE1. SUMMARY OF INFORMATION SECURITY IN HEALTH SECTOR

\section{B. Objective of the research}

The general objective of the study is to assess patients' perception of health information security and its implication in some selected public and private hospitals in Addis Ababa. Thus in the effort of addressing the general objective, the research responds to the following research question.

- What is the level of patients' perception of health information security?

- What factors influences patient's perception?

- What is the implication of the current status of patients' perception on information security at hospitals? 


\section{Rationale and significance of the research}

Information security per se has drawn significant attention among mainstream information systems scholars, yet very little has been published in the mainstream information systems journals on information security in healthcare (Appari and Johnson, 2008). Lack of proper information security and protection of privacy could lead to failure in health care delivery and management, and there by endangering societal rights to get health care. Safeguards to individual health care information are imperative to preserve the health care delivery relationship and the integrity of the patient attitude and perception towards the service and their record. To the knowledge of the researcher, there were no empirical studies in Ethiopian context on patients' perception of health information security at public or private hospitals. Thus through this study attempt is being made to contribute to the body of knowledge in the area by empirically test patent's perception towards health information security.

As part of continuous effort in the area, this study contributes to the existing literature by providing additional dimension of health information security in developing economies like Ethiopia. Furthermore this research will contribute to the effort of improving the quality of life in general by supporting the effort of creating conducive environment for secured communication between patients and physicians. It is also worth mentioning that, as information is a key resource nowadays, an effort and means to secure this resource will be considered as an important ingredient in the effort of facilitating socio-economic development. The results of the study will also be used by policy makers to include patients' concern, as they are the primary stakeholders of health sector, as an input in the formulation of policies and guidelines on health information security. Finally as this study is in one of the developing economies in Africa, it can also be seen as an additional contribution in the area with in different context.

\section{RESEARCH METHODOLOGY}

\section{A. Study Area}

The study is conducted in two public and two private hospitals in Addis Ababa, the capital of Ethiopia. According to Addis Ababa Health Bureau Report, there are 9 public hospitals and 17 general private hospitals serving a total population of over 3 million. For this study, as indicated above 4 hospitals namely Tikur Anbesa, Yekatit 12, Betezata, and St. Gebriel hospitals were selected considering their capacity in serving large number of patients and their proximity in access for a researcher.

\section{B. Source and Study population}

Patients generally can be categorized in to outpatients and inpatients. This study particularly focused on out patent's due to the assumption that they will be freer from bias in telling about the health centers. All patients attending general medical OutPatient Departments (OPD) in the hospitals were the source population. As to the inclusion criteria, all patients who have already seen a doctor/nurse in the hospitals' OPD during the study period and those who are 18 years old and above are included in the study. With regard to exclusion criteria, patients whose age is less than 18 years old, have communication problem and unconscious were excluded from the study.

\section{Sampling Techniques and Sample Size}

Study subjects were selected using convenience sampling method. Accordingly 200 patients who came to the general medical OPD at the selected hospitals were approached immediately as they take a seat in a waiting room before and after encountering a physician. At this point, patients who had an experience of visit of a physician were identified by asking the respondents themselves. Based on the inclusion criteria, all eligible patients who came for follow up and treatment were approached to fill an interviewer administered questionnaire. Patients were approached consecutively until the required sample size of 50 is reached from each hospital.

\section{Data Collection instrument and method}

In the effort of addressing research questions posed, a questionnaire with detailed items is developed. The survey instruments are constructed using the literature pertaining to the constructs. The process follows from an exhaustive review of the literature to derive multi-item scales for the constructs. Wherever possible, existing questionnaire items with previously established levels of reliability and validity are adopted to measure the variables.

With respect to data collection process one data collector, who had diploma, was selected and given training by lecture and role play method (on the objective of the study, method of administering questionnaires, confidentiality and way of handling and approaching study subjects). This was just to facilitate the data collection task as the time was short. The data is collected from 200 patients on follow up and treatment in the general medical OPDs of the hospitals. After explaining the objective of the study, informed consent was obtained from the patients and interviewer administered questionnaire was used to collect the data. To add on the data quality and reliability by helping patients better understand the questions, the questionnaire is translated into Amharic, Ethiopian national language. And it is pre-tested on 15 patients who were not included in the actual study. This was done to check whether there is common understanding between the data collectors and patients. And it enabled me to ensure the consistency and completeness of the questionnaire. After doing this, minor corrections was made on the questionnaire.

\section{E. Data processing and analysis}

Collected data was analyzed using the statistical package for social scientists (SPSS) version 15.0. Tables, graphs and cross tabulations was used to present different statistical analysis. With regard to the procedure, questionnaire items were firsts coded using the said analysis software, which was followed by inputting and exploring the data for understanding. After having the data in the required format conducting different statistical analysis shown in the result section of this paper was done. 


\section{F. Variables}

Independent demographic Variables studied variables

includes, socio such as gender, age, marital status, occupation, and educational level, patients' reported previous knowledge/thought about security, patients' expectation of problems by disclosing health information, perceived threat due to security breach, level of security of information given to a physician. In addition

patients' care for security, knowledge of national information security policy, knowledge of hospital security policy, confidentiality of information in medical records, security of examination room, over all patient knowledge about information security in hospitals, past experience of problem of security breach are also considered. The two important dependent variables assumed to be affected by the independent variables listed above were patients' perception of health information security and its implication.

\section{G. Ethical Considerations}

Various approaches were considered to address ethical issues. Written letter of permission is obtained from Faculty of Informatics and Addis Ababa Health Bureau. The purpose and objective of the study and the confidentiality of their response was clearly explained for the participants. Finally, patients were involved only after informed consent was obtained. It is also worth mentioning that respondents were also being informed that it is their right to participate or not to participate and their involvement will not have any thing to do with their treatment.

\section{H. Dissemination of results}

Finally, though the major outlets for the dissemination of the result of this research out put for the general public are conferences and journals in the area, Ministry of Health, Addis Ababa Health Bureau, and Addis Ababa University are also other responsible bodies at a national and regional level to make use of the research outputs in the health sector.

\section{ANALYSIS AND RESULT}

This section of my research report presents my major findings from the literatures review and empirical data analysis collected from the study area selected. I enrolled 50 patients from each of the 4 hospitals ( 2 public and 2 private) with a total of 200 patients.

\section{A. Demographic and Socio-Economic Data}

The demographic and socio-economic data presented in Table 1, contains variables that are assumed to have a positive or negative impact on the issue raised. As it can be seen from the attached questionnaire, it includes gender, age, educational level, occupation, average income and martial status. Accordingly over all, there were 108 females and 92 males. It is presented below against the age category of research participants.
TABle 1: DEMOGRAPHIC AND SOCIO-ECONOMIC DATA

\begin{tabular}{|c|c|c|c|c|c|c|c|}
\hline \multirow[b]{2}{*}{ Age } & \multirow{2}{*}{\multicolumn{2}{|c|}{ نั }} & \multicolumn{5}{|c|}{ Educational Level; } \\
\hline & & & $\begin{array}{c}\text { No } \\
\text { Edu } \\
\end{array}$ & $1-8$ & $9-12$ & $\begin{array}{c}\text { Dipd } \\
\text { Degr }\end{array}$ & $\begin{array}{l}M . S \\
\text { c\&a } \\
\text { bove }\end{array}$ \\
\hline \multirow{2}{*}{$18-30$} & M & 29 & 0 & 1 & 13 & 18 & 2 \\
\hline & $\mathrm{F}$ & 41 & 0 & 1 & 15 & 16 & 1 \\
\hline \multirow{2}{*}{$31-45$} & M & 16 & 1 & 4 & 5 & 24 & 4 \\
\hline & $\mathrm{F}$ & 34 & 2 & 9 & 6 & 22 & 3 \\
\hline \multirow{2}{*}{$>45$} & M & 47 & 4 & 7 & 17 & 3 & 1 \\
\hline & $F$ & 33 & 5 & 4 & 10 & 1 & 1 \\
\hline \multicolumn{2}{|c|}{ Total } & 200 & 12 & 26 & 66 & 84 & 12 \\
\hline
\end{tabular}

Another observation was on the patient's preference of hospitals as evident in Table 2. Majority of the study subjects whose age is 45 years and above went to public hospitals and those with in the age range of $31-45$ years to the private hospitals respectively.

TABLE 2: PATIENTS PREFERENCE OF HOSPITALS ALONG WITH THEIR AGE

\begin{tabular}{|c|r|r|r|r|}
\hline & \multicolumn{4}{|c|}{ Name of The Hospital } \\
\cline { 2 - 5 } Age & $\begin{array}{c}\text { Yekatit12 } \\
\text { (Public) }\end{array}$ & $\begin{array}{c}\text { Tikur } \\
\text { Anbesa } \\
\text { (Public) }\end{array}$ & $\begin{array}{c}\text { BetaZata } \\
\text { (Private) }\end{array}$ & $\begin{array}{c}\text { SaintGeb } \\
\text { (Private) }\end{array}$ \\
\hline $18-30$ & 30 & 0 & 20 & 20 \\
\hline $31-45$ & 0 & 10 & 20 & 20 \\
\hline$>45$ & 20 & 50 & 10 & 0 \\
\hline
\end{tabular}

With respect to the educational level of the respondents, significant portion of study subjects were at a high school and first degree or diploma level. The demographic data of this study also shows that as the educational status of the patient's increases, most of them go to the private hospitals. A detail of respondent's educational level is shown in Table 1 .

As to the marital status of patient respondent's, majority of them $(48 \%)$ were married while significant number of them $42 \%$ and $9 \%$ were single and divorced respectively. It is also worth mentioning that patients who run private business and employees count for $40 \%$ each from the total respondents while the rest $15 \%$ and $5 \%$ were students and jobless.

\section{B. Patent's Perception of Health Information Security}

As indicated in the previous sections of this study, a number of variables were identified in measuring patient's perception of information security. Accordingly, they were asked if they were aware of health information security in general. Their response shows that the younger a patient is the more awareness he/she has about health information security. And this is shown in Table 3. 
TABle 3: Patients AwARENESS IN GENERAL

\begin{tabular}{|c|c|c|c|c|c|}
\hline \multirow[b]{3}{*}{ Age } & \multicolumn{5}{|c|}{ Awareness } \\
\hline & StrAgree & Agree & Indifferent & Disagree & $\begin{array}{c}\text { StrDis } \\
\text { agree }\end{array}$ \\
\hline & Count & Count & Count & Count & Count \\
\hline $18-30$ & 22 & 20 & 2 & 14 & 12 \\
\hline $31-45$ & 7 & 10 & 0 & 26 & 7 \\
\hline$>45$ & 14 & 3 & 20 & 12 & 31 \\
\hline
\end{tabular}

Similarly patients were asked if they consider patients medical record as confidential communication between the doctor or other health professional and the patient. In line with this, of all 200 respondents 104 (above 50\%) agree that the communication should be confidential while the rest 36 and 60 were against the idea and indifferent respectively. The result also reveals that, their educational level and their perception about the confidentiality of communication between them and their doctors have a positive relation. For instance 45 out of 84 respondents who have either diploma or $1^{\text {st }}$ degree agree that the said communication should be confidential, while only 3 respondents out of 12 agree on the confidentiality issue raised. The detail is shown in Table 4.

TABLE 4: PATIENTS PERCEPTION ON CONFIDENTIALITY OF COMMUNICATIONS

EduLevel * Confidentiality Crosstabulation

\begin{tabular}{|c|c|c|c|c|c|c|}
\hline \multicolumn{7}{|l|}{ Count } \\
\hline & \multicolumn{5}{|c|}{ Confidentiality } & \multirow[b]{2}{*}{ Total } \\
\hline & ptrAgree & Agree & hdifferen & Disagreet & trDisagre & \\
\hline EduLeı NoEdu & 3 & 0 & 0 & 6 & 3 & 12 \\
\hline $1-8$ & 15 & 0 & 9 & 2 & 0 & 26 \\
\hline $9-12$ & 4 & 32 & 27 & 3 & 0 & 66 \\
\hline DipAndDe & 40 & 5 & 18 & 21 & 0 & 84 \\
\hline MScAndA & 1 & 4 & 6 & 1 & 0 & 12 \\
\hline Total & 63 & 41 & 60 & 33 & 3 & 200 \\
\hline
\end{tabular}

In the effort of measuring patients perception of information security, the next question posed was whether disclosing medical record for third party will create a problem on them or not. In responding to this question, $85 \%$ of the respondents either agree or strongly agree with the idea that disclosing medical records will create a problem on personal life of the patient. The rest $15 \%$ were indifferent on the issue raised.

Another item used to measure patients perception was, a question about their belief on the security of different section of the health institution including, the examination room. The result reveals that $55 \%$ of the respondents believe that different sections of the hospital are not secured while $15 \%$ and $30 \%$ of them are indifferent and against the idea rose respectively. In order to crosscheck their response attempt has also been made to see the relationship between their information security awareness and the value that they give for their medical record security at the hospitals. In connection to this, as it can be seen from the table 5 below the more patients are aware about security issues the more they will concerned about their medical records' security.
TABLE 5: AWARENESS AND PATIENTS FEELING ABOUT SECTIONS SECURITY AT HOSPITALS

Awarness * SecuConc Crosstabulation

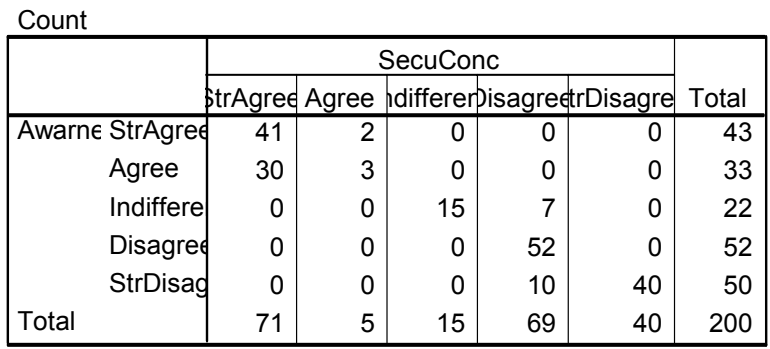

As it can be seen from the Table 5, out of 43 respondents who strongly express their awareness on health information security, 41 (more than 95\%) study subjects were very much concerned about their medical records. On the other hand out of 69 respondents who reported that they are not concerned about their medical records, there were only 7 who were indifferent in their general awareness about health information security, the rest being with no expressed awareness.

Patients trust on health institutions was another variable used to assess patient's perception. Accordingly as evident from Figure 2, for a reversely coded item on trust $40 \%$ in total reported that they do not trust health institutions while $30 \%$ and other $30 \%$ do not agree with that and being indifferent respectively.

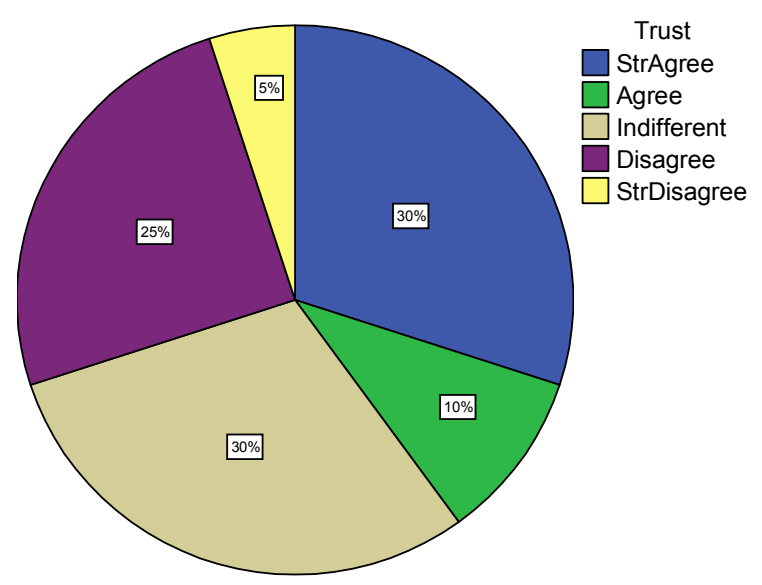

FiguRE 2: PATENTS TRUST ON HEALTH INSTITUTIONS

Experience of problems in the past, because of insecurity of personal health information, was still another indicator of their perception. And the response shows that, $20 \%$ of the respondents have had such bad experience due to insecurity of data while other $20 \%$ and $60 \%$ were not in that position to experience such a problem and indifferent respectively. Patients were also asked if a third party legal entity can inquire their medical records and their response reveals that $70 \%$ of the respondents either agree or strongly agree while the rest $30 \%$ are indifferent about the issue.

It also worth mentioning that significant number of patients responded that they are not familiar with any written 
or oral regulations and procedures regarding health information security both at a national and institutional level.

In line with very poor awareness about any formal or informal security procedures, responses of patients also reveals that there is no as such inquiring medical records culture for any reason. With this respect, $75 \%$ of the respondents indicate that they didn't inquire their medical record so far while $21 \%$ and $4 \%$ were inquiring and were not sure respectively.

\section{DISCUSSIONS}

Thus from the empirical tests using the questionnaire items, patients perception of information security at the public and private hospitals selected is generally poor. This is shown by a response of less concern for information security by almost $45 \%$ of study subject for the question about security concern at the health institutions. Their response of lack of trust on health institutions, (40\%), also exhibits the same thing. It is also apparent from the results that their perception has some correlation with their educational background and awareness about it. This can be interpreted as the more people are informed the more they may be concerned on issues.

Literatures identified various factors affecting patient's perception. Through this research educational background, past experience on security breach, and their general awareness about security were identified as important factors in affecting patents perception. This is exhibited on the result section as; above $50 \%$ of the respondents believe that confidentiality of communication between patients and physicians is important. It also worth mentioning that over $95 \%$ of respondents who reported that they have general security awareness shown strong concern on the security of their medical records. This result is in line with the study on the Impact of Personal Dispositions on Privacy and Trust in Disclosing Health Information Online made by Bansal et al. (2007).

From both the literature review and empirical tests conducted through out this research it is easy to learn the implications of patient's perception on different aspects of the health sector. Considering the sensitive nature of the medical records of individuals, patient's perception about its security will impact the services delivery and user satisfaction. And this is in line with the study on Patient privacy and confidentiality conducted by Chalmers and Muir (2003).

Improper disclosure of health information can disturb conducive environment expected between patients and the health sectors. This is because the efficacy of the healthcare relationship depends on the patient's understanding that the information recorded by a physician will not be disclosed. As shown in the result section $55 \%$ of the respondents believe that different section of the hospitals that they visited are not secured. In such a scenario many patients might refuse to provide physicians with certain types of information needed to render appropriate care if patients do not believe that information would remain confidential. This in turn will obviously is limiting the level of services that patients as a citizen should get from health sectors.

\section{CONCLUSION}

As evident from our research, information security in general and health information security in particular needs even more attention. As part of various efforts in the area, this study investigates and found that patient's perception in selected public and private health institutions is poor. Attempt has also been made to identify factors associated with it and implications it has on the health sector as a whole.

In line with various supporting literatures, considering the sensitivity nature of health information any party involved in any task related with this health information should be trustworthy, protect patient health information, and adhere to security principles. Another equally important task should be to enable patients develop awareness and good perception on the security of their medical records.

\section{REFERENCES}

1. Appari, A. and Johnson M. (2008) Information Security and privacy in health care: Current State of Research.

2. Addis Ababa Health Bureau Report. List of Health Facilities in Addis Ababa. 2009

3. Angst, C.M., Agrawal, R., and Downing, J. (2006) -An Empirical Examination of the Importance of Defining the PHR for Research and for Practice, Robert H. Smith School Research Paper No. RHS06-011 Available at SSRN: http://ssrn.com/abstract=904611

4. Bansal, G., Zaheid, F.,M., Gefen, D. (2007) - The Impact of Personal Dispositions on Privacy and Trust in Disclosing Health Information Online, Americas Conference on Information Systems

5. Chalmers and Muir (2003) - Patient privacy and confidentiality. BMJ Volume 326, 2003

6. Campbell, B., Thomson, H., Slater, J., Coward, C., Wyatt, K., and Sweeney, K.( 2007 ) -Extracting Information from Hospital Records: What Patients Think About Consent, Quality and Safety in Healthcare, vol. 16, no. 6, pp 404-408

7. Jim Chalmers and Rod Muir. Patient privacy and confidentiality. British Medical Journal. 2003; 326; 725-726.

8. Legislative Reference Bureau. Convention for the Protection of Individuals With Regard to Automatic Processing of Personal Data. State Capitol Honolulu, Hawaii, 2000.

9. Mercuri, R.T. (2004) -The HIPAA-potamus in Health Care Data Security, Communications of the ACM, vol.47, no.7

10. NRC National Research Council (1997) - For the Record: Protecting Electronic Health Information.

11. Sankar, P., Moran, S., Merz, J.F., Jones, N.L. (2003) - Patient Perspectives on Medical Confidentiality: A Review of the Literature, Journal of General Internal Medicine, vol.18, pp 659-669

12. Willison D.J. et al. Patients' consent preferences for research uses of information in electronic medical records. British Medical Journal. 2003, 326: 373. 